Communication

\title{
In vitro Electroporation in the Presence of CRISPR/Cas9 Reagents as a Safe and Effective Method for Producing Biallelic Knock-Out Porcine Embryos
}

\author{
Masahiro Sato ${ }^{1,}{ }^{*}$, Hansol Jin ${ }^{2}$, Eri Akasaka ${ }^{1}$, Kazuchika Miyoshi ${ }^{2,3}$
}

1. Section of Gene Expression Regulation, Frontier Science Research Center, Kagoshima University, Kagoshima 890-8544, Japan; E-Mail: masasato@m.kufm.kagoshima-u.ac.jp; stylistics777@yahoo.co.jp

2. Laboratory of Animal Reproduction, Faculty of Agriculture, Kagoshima University, Kagoshima 890-0065, Japan; E-Mails: jinhansol73@gmail.com; kmiyoshi@agri.kagoshima-u.ac.jp

3. The United Graduate School of Agricultural Sciences, Kagoshima University, Kagoshima 890-0065, Japan

* Correspondence: Masahiro Sato; E-Mail: masasato@m.kufm.kagoshima-u.ac.jp

Academic Editor: Joep Geraedts

Special Issue: Genome Editing

OBM Genetics

2021, volume 5, issue 1

doi:10.21926/obm.genet. 2101123
Received: September 01, 2020

Accepted: February 01, 2021

Published: February 18, 2021

\begin{abstract}
The production of genetically modified (GM) pigs is considered valuable in biomedical research for the development of model animals for various diseases and pigs with resistance against viral infection. The porcine genome may be modified using several methods, such as somatic cell nuclear transfer (SCNT) using GM cells as the SCNT donor, direct injection of the transgene or the genome editing components (GEC) into fertilized eggs referred to as zygotes, the in vitro electroporation (EP) of the zygotes in the presence of GECs, viral infection using retroviruses, injection of the GECs into the SCNT-treated embryos, and the in vitro EP of the SCNT-treated embryos in the presence of GECs. In our previous study, we administered a cytoplasmic injection of CRISPR/Cas9-based GEC into parthenogenetically-activated porcine oocytes (referred to as parthenotes) and observed that these oocytes comprised a mixture of
\end{abstract}

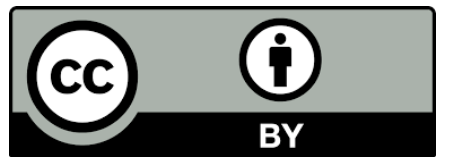

(C) 2021 by the author. This is an open access article distributed under the conditions of the Creative Commons by Attribution License, which permits unrestricted use, distribution, and reproduction in any medium or format, provided the original work is correctly cited. 
genome-edited and genome-unedited cells, referred to as the "mosaic". In contrast, when in vitro EP of the SCNT-treated embryos in the presence of GEC was performed, bi-allelic knock out (KO) of the target gene was detected in most oocytes $(82 \% ; 9 / 11)$. The production of biallelic KO piglets is particularly beneficial for investigating GM domestic animals as it does not require further breeding trials to obtain bi-allelic KO individuals, which would otherwise be a time-consuming and laborious task. In this context, the present study was aimed to confirm the efficiency of in vitro EP in producing bi-allelic KO porcine embryos without multiple breeding trials, for which parthenotes were subjected to EP in the presence of a ribonucleoprotein containing Cas9 protein and single-guide RNA (targeted toward GGTA1). The treated embryos were cultured until they transformed into blastocysts. The genomic DNA isolated from these blastocysts was used for molecular biology analysis to detect the possible insertion and deletion of sequences (indels) at the GGTA1 locus. Among the 32 blastocysts obtained, 21 (66\%) were observed to be the bi-allelic KO ones. The remaining embryos either had a normal phenotype $(25 \% ; 8 / 32)$ or mosaic mutations $(9 \% ; 3 / 32)$. These findings confirm the efficiency of in vitro EP in producing bi-allelic KO porcine embryos.

\section{Keywords}

Electroporation; $\alpha$-1,3-galactosyltransferase; CRISPR/Cas9; ribonucleoprotein; single guide RNA; mosaicism; Bi-allelic KO; indels; porcine parthenotes

\section{Introduction}

Recently, a series of nuclease-based genome-editing tools and technologies have been developed, including zinc-finger nuclease (ZFN), transcription activator-like effector nucleases (TALEN), and clustered regularly-interspaced short palindromic repeats (CRISPR)/CRISPR-associated (Cas)9 (CRISPR/Cas9), etc., which enable targeted and efficient modification of various eukaryotic species, including mammals [1, 2]. The CRISPR/Cas9-based genome editing requires a guide RNA (gRNA) that would bind to the specific chromosomal DNA site together with Cas9 endonuclease [36]. Once bound, each of the two independent nuclease domains in Cas 9 cleaves a DNA strand three bases upstream of the protospacer adjacent motif (PAM) and introduces double-strand breaks (DSBs) at the host chromosome target site, which are later repaired through non-homologous endjoining (NHEJ). The NHEJ-based repair process introduces nucleotide insertions or deletions (indel mutations), which may lead to the formation of premature termination (stop) codons, thereby causing protein expression failure via nonsense-mediated mRNA decay, a translation-dependent surveillance mechanism in eukaryotes [7]. The simplicity and the convenience of gRNA designing have enabled the widespread application of CRISPR/Cas9 as a powerful tool for producing genetically modified (GM) organisms [1, 2].

Currently, several methods are available for the production of GM embryos and piglets, such as 1) microinjection of nucleic acids (NAs) into the pronuclei of zygotes [8]; 2) somatic cell nuclear transfer (SCNT) from a GM donor cell into the enucleated porcine oocytes [9-11]; 3) microinjection of nucleic acids (NAs) into SCNT-treated embryos [12]; 3) in vitro electroporation (EP) of porcine zygotes in the presence of NAs [13]; and 5) in vitro EP of the SCNT-treated embryos in the presence 
of NAs [14]. According to Sato et al. [14], in the case of the 61 genome-edited piglets produced since 2013, most of these experiments were focused either on using SCNT-based production of GM piglets or on zygote microinjection [14], while the in vitro EP-based production of GM piglets was used by only a few laboratories $[13,15-20]$. This is in contrast to the case of genome-edited mice. For instance, since Kaneko et al. [21] first reported the successful use of this technology in 2014, several genome-edited mice have been produced by numerous laboratories worldwide [22-34]. Tanihara et al. [13] pioneered in demonstrating in vitro EP as a promising tool for producing GM piglets with high efficiency. For instance, when in vitro EP (30 V, square pulses, $1.0 \mathrm{~ms}$ in duration, and repeated 5 times) using an electrode (LF501PT1-20; BEX, Tokyo, Japan) and CUY21EDIT II electroporator (BEX) in the presence of genome editing components (GECs) [Cas9 protein ( $50 \mathrm{ng} / \mu \mathrm{L})$ along with a single guide RNA (sgRNA) (200 ng/ $\mu \mathrm{L}$ )] was performed for in vitro fertilized (IVF) porcine eggs (13 $\mathrm{h}$ after IVF), successful genome editing was observed in $67 \%$ (10/15) of the treated embryos (blastocysts) [13]. The authors named this process "gene editing by electroporation of Cas9 protein (GEEP)". Since then, several genome-edited pigs have been produced using this GEEP technique, including the TP53 (which encodes p53)-mutant pigs [15], CD163 [a putative fusion receptor for the virus causing the porcine reproductive and respiratory syndrome (PRRS)]-mutant pigs [16], and myostatin-mutant pigs [20].

When producing genetically engineered domestic animals, the production of mutations (or transgene insertion) in both alleles of the gene is important as it enables evaluating the results of the phenotypic alteration caused by the gene alteration in an early stage of the investigation. In the event of the GM animals developing mutations (or transgene insertion) only in one allele, obtaining homozygous knock out (KO) animals is time-consuming (at least $>0.5$ years in the case of pigs) and cumbersome [35]. In mice, in vitro EP is beneficial for producing animals with bi-allelic KO phenotypes with relatively high efficiency, as the GECs are incorporated into the embryo with lesser bias [34]. This is in contrast with the findings of our previous work, in which a cytoplasmic injection administered to obtain genome-edited porcine embryos resulted in frequent mosaic mutations in the target locus [36]. Unfortunately, there is little information regarding the efficiency of the embryos with bi-allelic KO phenotype when the porcine zygotes or IVF-derived oocytes are subjected to in vitro EP in the presence of GECs.

In the present study, it was investigated whether in vitro EP would also be beneficial for the acquisition of embryos with bi-allelic KO phenotypes in the case when porcine parthenotes are used as an alternative to zygotes. Parthenotes were selected as it is convenient to obtain ovaries carrying the oocytes from a slaughterhouse without any additional cost, and the resulting in vitro activated oocytes (referred to as "parthenotes") mimic the development of zygotes, at least up to the early gestational stage [37]. In order to induce efficient genome editing in a target locus, Cas9 protein/sgRNA complex [referred to as ribonucleoprotein (RNP)] targeted toward GGTA1, a gene encoding $\alpha-1,3$-galactosyltransferase ( $\alpha$-GaIT), was used as it allows rapid genome editing without leaving any traits (GECs) in the target chromosomes [38]. Whether the porcine embryos have biallelic KO phenotype is easily detectable using the molecular biology analysis of the porcine genome DNA isolated from a single blastocyst. 


\section{Materials and Methods}

\subsection{Preparation of porcine parthenotes}

The porcine parthenotes were produced using the methods described in our previous studies [39]. Briefly, the ovaries collected from prepubertal gilts at a local slaughterhouse were transported to the laboratory, and the cumulus oocyte complexes (COCs) were extracted from the antral follicles with 2-mm to 5-mm diameter using an 18-gauge needle (Terumo, Tokyo, Japan) fixed to a 5-mL disposable syringe (Nipro, Osaka, Japan). The COCs were washed three times with HEPES (Nacalai Tesque, Kyoto, Japan)-buffered Tyrode's lactate-pyruvate-polyvinyl alcohol (PVA; Sigma-Aldrich Co., Saint Louis, MO, USA) (HEPES-TLP-PVA). Next, approximately 40-50 COCs were transferred to 200 $\mu \mathrm{L}$ of the maturation medium (90\% (v/v) TCM-199 containing Earle's salts (Gibco BRL, Grand Island, NY, USA) and supplemented with $0.91 \mathrm{mM}$ sodium pyruvate (Sigma-Aldrich Co.), $3.05 \mathrm{mM}$ D-glucose (Wako Pure Chemical, Osaka, Japan), $0.57 \mathrm{mM}$ cysteine hydrochloride hydrate (Sigma-Aldrich Co.), $10 \mathrm{ng} / \mathrm{mL}$ epidermal growth factor (Sigma-Aldrich Co.), $10 \mathrm{IU} / \mathrm{mL}$ eCG (Aska Pharmaceutical Co., Tokyo, Japan), $10 \mathrm{IU} / \mathrm{mL}$ hCG (Aska Pharmaceutical Co.), $100 \mu \mathrm{g} / \mathrm{mL}$ amikacin sulfate (Meiji Seika, Tokyo, Japan), $0.1 \%(w / v)$ PVA, and $10 \%(v / v)$ pig follicular fluid covered with paraffin oil (Nacalai Tesque) in a 35-mm dish (\#1008; Becton Dickinson, Franklin Lakes, NJ, USA), and then preequilibrated overnight at $38.5^{\circ} \mathrm{C}$ in $5 \% \mathrm{CO}_{2}$ atmosphere. After 42 to $44 \mathrm{~h}$ of maturation, the cumulus cells were removed by pipetting with $0.1 \%(w / v)$ hyaluronidase (Sigma-Aldrich Co.), and the oocytes with polar bodies were selected for further experiments.

The parthenote production was performed by placing the denuded oocytes (20-40) between two wire electrodes that were $1 \mathrm{~mm}$ apart in an activation medium [250.3 mM sorbitol, $0.5 \mathrm{mM}$ $\mathrm{Ca}\left(\mathrm{CH}_{3} \mathrm{COO}\right)_{2}, 0.5 \mathrm{mM} \mathrm{Mg}\left(\mathrm{CH}_{3} \mathrm{COO}\right)_{2}$, and $0.1 \%$ bovine serum albumin (BSA)] [40] and then inducing activation with one direct current pulse of $100 \mathrm{~V} / \mathrm{mm}$ for $50 \mu \mathrm{s}$ using an LF101 Fusion Machine (Nepa Gene Co., Chiba, Japan).

\subsection{Preparation of RNP}

The gRNA (also referred to as \#3 gRNA; [41]) capable of recognizing a 20 bp sequence spanning the translation initiation codon (ATG) upstream of the protospacer adjacent motif (PAM) sequence (AGG) on the $4^{\text {th }}$ exon of porcine GGTA1 (Figure 1C) was designed. The gRNA that ranked first in the CHOPCHOP analysis (https://chopchop.cbu.uib.no/), which is one of the most widely used web tools for CRISPR-based genome editing, was selected. Furthermore, our preliminary study already confirmed that there was no off-target induction in the parthenotes (blastocysts) microinjected with a mixture of CRISPR/Cas9-related GECs [36].

The Integrated DNA Technologies, Inc. (IDT; Coralville, Iowa, USA) synthesized the gRNA as an Alt-R ${ }^{\text {TM }}$ CRISPR crRNA product. The crRNA and tracrRNA (purchased from IDT) were combined for annealing to generate the sgRNA, which was followed by the addition of recombinant Cas9 protein (TaKaRa Bio, Inc., Ohtsu, Japan) to form the RNP, using the methods reported by Ohtsuka et al. [42]. The resulting RNP contained the humanized Cas 9 protein $(50 \mathrm{ng} / \mu \mathrm{L})$ and the sgRNA $(200 \mathrm{ng} / \mu \mathrm{L})$, as described by a previous report by Tanihara et al. [13]. 


\subsection{In vitro EP}

The EP was performed using the method described by Hashimoto and Takemoto [22]. An electroporation chamber (\#LF610P4-4_470; BEX Co. Ltd.) containing two platinum block electrodes situated at a distance of $1 \mathrm{~mm}$ from each other (Figure 1A) was placed under a stereoscopic microscope and then connected to an electric pulse generator CUY21EDITII Genome Editor ${ }^{\mathrm{TM}}$ (BEX Co. Ltd.). Approximately 30 parthenotes ( $6 \mathrm{~h}$ after activation) were added to a $5-\mu \mathrm{L}$ drop of OptiMEM (Invitrogen, Carlsbad, CA, USA) containing $2 \mu \mathrm{g} / \mu \mathrm{L}$ tetramethylrhodamine-dextran $3 \mathrm{kDa}$ (\#D3307; Thermo Fisher Scientific Inc., Waltham, MA, USA) placed between the electrodes (Figure 1A). The EP was performed under the following conditions: $30 \mathrm{~V}$, square pulses, $1.0 \mathrm{~ms}$ in duration, at 99 ms intervals, repeated seven times (only 4 times in certain cases). The EP-treated parthenotes were cultured in the PZM-3 (mPZM-3) medium [43] at $38.5{ }^{\circ} \mathrm{C}$ under $5 \% \mathrm{CO}_{2}: 5 \% \mathrm{O}_{2}: 90 \% \mathrm{~N}_{2}$ atmosphere for 2 days until the 2-cell stage was reached, following, which they were subjected to the evaluation of the uptake of fluorescent dye by the embryos.

In order to perform the EP with GECs, 10-20 parthenotes (6 $\mathrm{h}$ after activation) were added to a $5-\mu \mathrm{L}$ drop of Opti-MEM containing RNP and immediately subjected to in vitro EP under the conditions similar to those used for transfection with tetramethylrhodamine-dextran (3 kDa). After the EP, the embryos were cultivated in the PZM-3 medium for 7 days until blastocyst formation and then subjected to molecular biology analysis (to detect the possible mutations in the $4^{\text {th }}$ exon of GGTA1) as described below.

\subsection{Isolation of Single Blastocysts and Genomic DNA for Molecular Biology Analysis}

Genomic DNA isolated from a single blastocyst was analyzed for possible mutations at the individual-embryo level. Briefly, the single blastocyst was transferred to a drop (1 $\mu \mathrm{L})$ of $\mathrm{Ca}^{2+}$ and $\mathrm{Mg}^{2+}$-free Dulbecco's modified phosphate-buffered saline (PBS) in a 0.5-mL PCR tube (\#PCR-05-C; AxyGen Scientific, Inc., Union City, CA, USA) using a mouth-controlled micropipette.

Genomic DNA was extracted from this single blastocyte by adding $20 \mu \mathrm{L}$ of lysis buffer $(0.125$ $\mu \mathrm{g} / \mathrm{mL}$ of proteinase $\mathrm{K}, 0.125 \mu \mathrm{g} / \mathrm{mL}$ of Pronase $\mathrm{E}, 0.32 \mathrm{M}$ sucrose, $10 \mathrm{mM}$ Tris- $\mathrm{HCl}(\mathrm{pH}$ 7.5), $5 \mathrm{mM}$ $\mathrm{MgCl}_{2}$, and $1 \%(\mathrm{v} / \mathrm{v})$ Triton $\left.\mathrm{X}-100\right)$ to the drop containing the blastocyst followed by incubation at $37^{\circ} \mathrm{C}$ for 2-3 days and then phenol/chloroform extraction. The resultant supernatant was subjected to ethanol-precipitation of DNA using a GenTLE ${ }^{\circledR}$ Precipitation Carrier (\#9094; TaKaRa Bio, Inc.). The precipitated DNA was dissolved in $20 \mu \mathrm{L}$ of sterile water and then stored at $4{ }^{\circ} \mathrm{C}$ until use.

In order to amplify the total genomic DNA, WGA was performed using the illustra GenomiPhi V2 DNA Amplification Kit (\#25-6600-31; GE Health Care Japan, Tokyo, Japan) as described in a previous report [44]. Briefly, $2 \mu \mathrm{L}$ of the extracted genomic DNA was mixed with $8 \mu \mathrm{L}$ of the reaction buffer containing $20 \mu \mathrm{L}$ enzyme, followed by overnight incubation at $30^{\circ} \mathrm{C}$.

The resulting WGA products $(2 \mu \mathrm{L})$ were subjected to the $1^{\text {st }}$ round of PCR using the Ex-S $\left(5^{\prime}\right.$ GCAAATTAAGGTAGAACGCA-3') and Ex-RV (5'-GCTGCCCCTGAGCCACAACG-3') primer set (Figure 1C), in a reaction volume of $20 \mu \mathrm{L}$ and under the PCR conditions described in a previous report [44]. Subsequently, $2 \mu \mathrm{L}$ of the $1^{\text {st }} \mathrm{PCR}$ products were subjected to nested PCR, performed using Ex4-2S (5'-CTCCTTAGCGCTCGTTGGCT-3') and Ex4-2RV (5'- GCAACTCTCTGGAATGCTTT-3') primer set (Figure $1 C)$, in a reaction volume of $20 \mu \mathrm{L}$ and under the same PCR conditions as used in the $1^{\text {st }}$ PCR round. The resultant product was $\sim 350 \mathrm{bp}$ in length, as determined by electrophoresis performed by running $1 \mu \mathrm{L}$ of the PCR product in $2 \%$ agarose gel to assess the band size. The remaining volume of 
the PCR product was subjected to purification using a NucleoSpin ${ }^{\circledR}$ Gel and PCR Clean-up (\#U0609A; TaKaRa Bio, Inc.).

The sequencing was performed by subjecting $4 \mu \mathrm{L}$ of the purified solution containing the nested PCR products, $\sim 350 \mathrm{bp}$ in length, to direct sequencing using the Ex4-2S primer, while a few samples of the nested PCR products were sub-cloned into the TA cloning vector pCR2.1 (Invitrogen) and the resulting clones were sequenced using the Ex4-2S primer. The direct sequencing of the PCR products was performed using a custom DNA sequencing service (Eurofins Genomics K.K., Tokyo, Japan).

\subsection{Fluorescence Observation}

The electroporated embryos were examined for tetramethylrhodamine-derived red fluorescence under a fluorescence microscope (BX60; Olympus, Tokyo, Japan) using DM600 filters (BP545-580 and BA6101F; Olympus). The micrographs were obtained using a digital camera (FUJIX HC-300/OL; Fuji Film, Tokyo, Japan) attached to the fluorescence microscope, and the images were printed using Mitsubishi digital color printer (CP700DSA; Mitsubishi, Tokyo, Japan).

\section{Results and Discussion}

In order to allow the parthenogenetic development of a porcine oocyte, the oocytes were isolated from the ovary and subjected to electric activation [45] for $12 \mathrm{~h}$, following which the embryos were subjected to in vitro EP. In order to determine whether the in vitro EP system employed in the present study was effective in the transfection of porcine parthenotes as well, first, EP was performed in the presence of $2 \mu \mathrm{g} / \mu \mathrm{L}$ of tetramethylrhodamine-labeled dextran $3 \mathrm{kDa}$, which is used frequently as the indicator of successful gene delivery in certain laboratories $[21,42,44]$. The parthenotes (10-20) were added to a drop of placed between electrodes and then electroporated under the following conditions: $30 \mathrm{~V}$, square pulses, $1.0 \mathrm{~ms}$ in duration, at $99 \mathrm{~ms}$ intervals, repeated seven times (Figure 1A). Among 31 parthenotes, 26 (84\%) exhibited red fluorescence in their cytoplasm (indicated by arrows in Figure 1B-a and 1B-b) when the 4-cell stage embryos were examined 2 days after the in vitro EP. No appreciable fluorescence was noted in the rest of these embryos (demarcated by an arrowhead in Figure 1B-a and 1B-b), indicating a failure in the uptake of dextran. Furthermore, all the untreated intact parthenotes were non-fluorescent (Figure 1B-c and 1B-d). These results indicated the significance of the in vitro EP system used in the present study. 
A

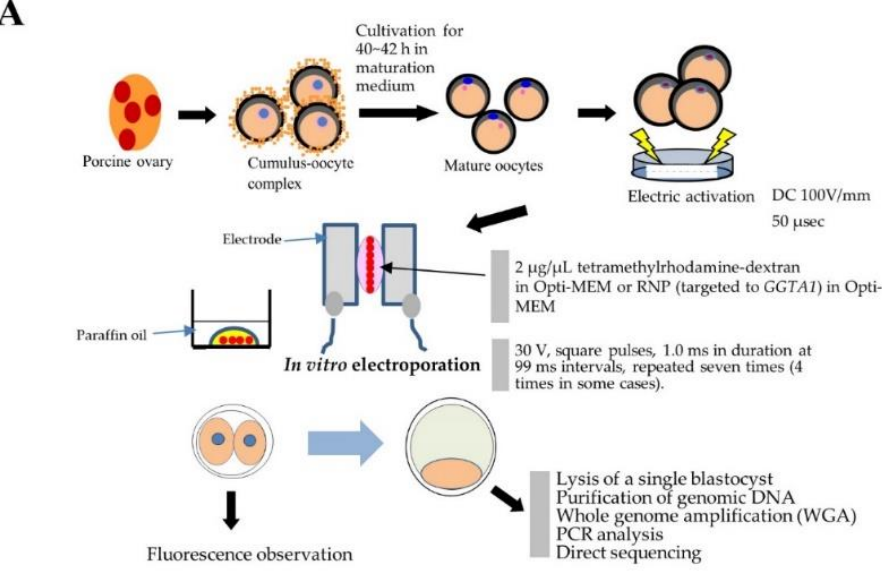

B

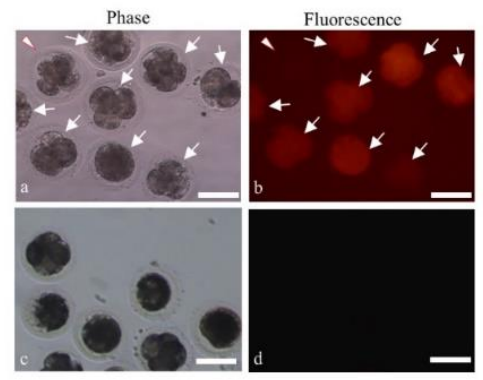

C

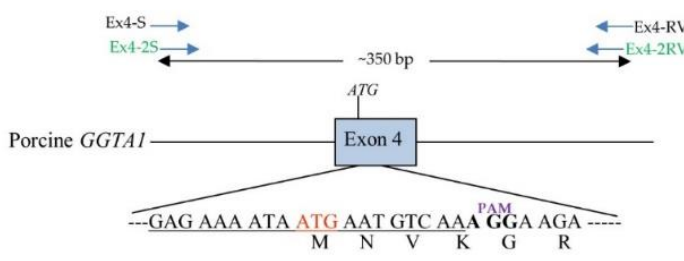

Figure 1 In vitro electroporation (EP) is beneficial for the acquisition of genome-edited porcine parthenotes. A. Schema for the in vitro EP of porcine parthenotes in the presence of tetramethylrhodamine-labeled dextran $3 \mathrm{kDa}$ or ribonucleoprotein (RNP). The ovaries collected from a slaughterhouse were used for isolating the cumulusoocyte-complex, which was further processed to obtain mature oocytes. After electric activation, these mature oocytes (parthenotes) were subjected to in vitro EP. The treated parthenotes were cultured until blastocyst formation in a drop of -3 medium under paraffin oil. The embryos at the $2 \sim 4$ cell stage were examined for fluorescence under a fluorescence microscope. Single blastocysts were used for isolating the genomic DNA and the subsequent molecular biology analysis. B. Fluorescence in the $2 \sim 4$ cellstage embryos after the in vitro EP in the presence of tetramethylrhodamine-labeled dextran $3 \mathrm{kDa}(\mathrm{a}, \mathrm{b})$ and that in the untreated embryos $(\mathrm{c}, \mathrm{d})$. Interestingly, over $80 \%$ of the parthenotes exhibited fluorescence (indicated by arrows in $a, b$ ), while the remaining embryos exhibited no fluorescence (indicated by arrowhead in $a, b)$. The untreated embryos did not exhibit any overt signs of fluorescence (c,d). Scale bar: $100 \mu \mathrm{m}$. C. Structure of exon 4 of porcine GGTA1. The sequence recognized by the sgRNA is underlined. ATG indicates the translation initiation site, and the amino acid sequence is depicted below the nucleotide sequence and the primer sets used (Ex4-S/Ex4-RV for the $1^{\text {st }}$ PCR and Ex4-2S/Ex4-2RV for the nested PCR) are depicted above the sequence. AGG (shown in bold) indicates the PAM site. 
Next, it was investigated whether effective genome-editing could be conducted on the endogenous GGTA1 via the electroporation of porcine parthenotes in the presence of RNP targeted toward GGTA1. The RNP contained the humanized Cas9 protein $(50 \mathrm{ng} / \mu \mathrm{L})$ and the sgRNA (200 $\mathrm{ng} / \mu \mathrm{L}$ ) targeted toward the 20-bp sequence immediately upstream of PAM on the exon 4 of GGTA1 (Figure 1C). Approximately 10-20 parthenotes were added to a drop of placed between electrodes and then electroporated under the conditions stated above. In this case, the in vitro EP was performed under the electric conditions of $30 \mathrm{~V}$ and $1.0 \mathrm{~ms}$ in the length of a square pulse with 99ms intervals (1.0). Pulse stimulation was repeated either 4 or 7 times. The treated embryos were subsequently transferred to the PZM-3 medium [43], which allowed for in vitro development for seven days until the blastocyst stage. The rate of development of the parthenotes to the 2-cell stage was $68 \%$ (34/50) when the pulse was administered 7 times. A similar rate (70\%; 35/50) was observed when the pulse stimulation was repeated 4 times (Table 1). In both the groups, the developmental rates for the parthenotes that reached the blastocyst stage ranged between 30\% (15/50) and 38\% $(19 / 50)$, which were slightly lower than the developmental rate observed for the untreated parthenotes (51\%; 46/91) (Table 1).

Table 1 Summary of the developmental rates to blastocyst stage after EP ${ }^{1}$ of porcine parthenotes.

\begin{tabular}{lllll}
\hline $\begin{array}{l}\text { Times of pulse } \\
\text { stimulation }\end{array}$ & No. of experiments & $\begin{array}{l}\text { Total number of } \\
\text { parthenotes examined }\end{array}$ & $\begin{array}{l}\text { No. of embryos } \\
\text { cleaved to the } \\
\text { 2-cell stage (\%) }\end{array}$ & $\begin{array}{l}\text { No. of embryos } \\
\text { developed to } \\
\text { blastocysts (\%) }\end{array}$ \\
\hline- & 3 & 91 & $73(80)$ & $46(51)$ \\
4 & 2 & 50 & $35(70)$ & $19(38)$ \\
7 & 2 & 50 & $34(68)$ & $15(30)$ \\
\hline
\end{tabular}

${ }^{1} \mathrm{EP}$ in the presence of ribonucleoprotein (RNP) is performed on the porcine parthenotes $6 \mathrm{~h}$ after the activation. The EP-treated embryos were subsequently cultured for seven days to the blastocyst stage and analyzed for the presence of mutations in the target gene at the molecular biology level.

${ }^{2} \mathrm{EP}$ was performed under the electric conditions of $30 \mathrm{~V}$ in voltage, $1.0 \mathrm{~ms}$ in the length of a square pulse, with 99-ms intervals (1.0), and pulse stimulation repeated 4 or 7 times using an electroporation chamber (\#LF610P4-4_470; BEX Co. Ltd.) connected to an electric pulse generator (CUY21EDIT II Genome Editor ${ }^{\mathrm{TM}}$, BEX Co. Ltd.). As the control, intact parthenotes without in vitro EP were cultivated until the blastocyst stage.

In order to determine the degree of genome editing at a molecular biology level, each blastocyst was subjected to genomic DNA isolation, followed by whole genome amplification (WGA) and the subsequent PCR of a region spanning the target sequence (Figure $1 \mathrm{C}$ ). Among the 34 blastocysts obtained, two blastocysts exhibited failed target region amplification. Therefore, it was decided to directly sequence the remaining 32 PCR products using the primer Ex4-2S as a nested sense primer (refer to Figure 1C; Materials and Methods section). Consequently, 21 samples (66\%) were observed to have bi-allelic KO phenotypes, as evidenced by no significant overlapping in the electrophoretograms of these samples (Table S1). A typical example of the results obtained from the direct sequencing of the nested PCR products using the Ex4-2S primer is presented in Figure 2- 
b (for embryo \#2) and 2-c (for embryo \#6), which depict the samples with nucleotide insertion and nucleotide deletion, respectively. The bi-allelic mutation in these samples was confirmed by subcloning the PCR products into PTA cloning vectors. All the obtained sub-clones ( 6 clones examined for each embryo) exhibited the same sequence as their respective parental products (Table S2).

The remaining embryos either had a normal phenotype (22\%; 7/32; Figure 2-a; exemplified by embryo \#5; Suppl. Table 1) or mosaic mutations (9\%; 3/32; Figure 2-d for embryo \#22; Suppl. Table 1 ). In the case of the mosaic mutations identified in embryo \#22, the sequence recognized by the sgRNA was overlapped and the nucleotide(s) were often displayed as " $\mathrm{N}$ ", indicating a mixture of edited (in the form of bi-allelic or mono-allelic KO mutation) and unedited cells (indicated by arrowheads in sample \#22 depicted in Figure 2-d; samples \#1, \#22, and \#32 in Table S1). In order to analyze the results of the DNA sequence analysis for samples \#1,\#22, and \#32 in further detail, the PCR products were sub-cloned into pTA cloning vectors. Sample \#1 was observed to have a normal sequence $(11 \%, 1 / 9)$, 18-bp deletion (including ATG) $(78 \%, 7 / 9)$, and replaced nucleotide (A to C) immediately below the PAM $(11 \%, 1 / 9)$ (Table S2), suggesting that this sample had mosaic mutations (including multiple KO alleles). Samples \#22 and \#32 exhibited phenotypes similar to that of sample \#1 (Table S2).

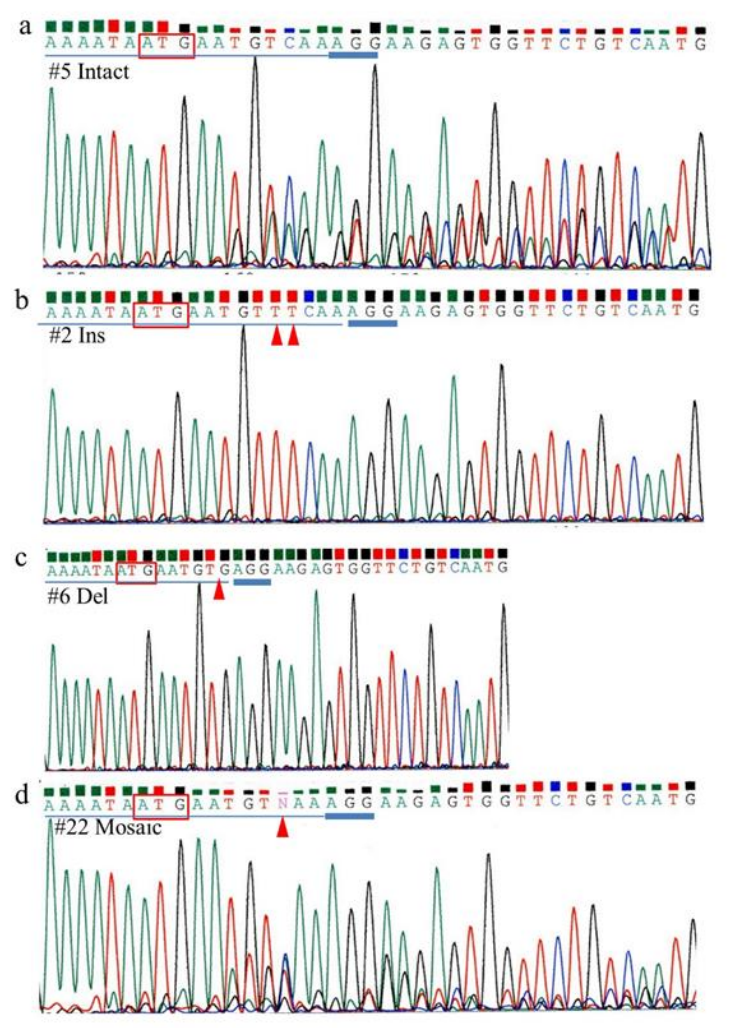

Figure 2 Ideogram pattern in the single blastocyst samples (presented in Suppl. Table 1) obtained after the direct sequencing of the nested PCR products using the Ex4-2S primer. a. Intact blastocyst (\#5). b. Blastocyst (\#2) exhibiting insertion (Ins). c. Blastocyst (\#6) exhibiting deletion (Del). d. Blastocyst (\#22) exhibiting mosaic mutation. ATG is depicted in red. The sequence recognized by the sgRNA is depicted with blue underlining. The PAM site is depicted with a bold blue line. The mutations are indicated by red arrowheads under the nucleotide sequence. 
In this study, in vitro EP was applied to obtain porcine embryos having a bi-allelic knockout (KO) phenotype with high efficiency. When the RNP targeted toward GGTA1 was used for the porcine parthenotes, $66 \%$ of the resulting blastocysts exhibited a bi-allelic KO phenotype, while the remaining embryos either had a normal phenotype (25\%) or mosaic mutations (9\%).

It is important to understand the reason for the generation of mosaic embryos after transfection with CRISPR/Cas9 components. Several studies have reported that the zygote-derived embryos injected with exogenous materials are often edited in a mosaic pattern [36, 42, 46, 47]. As mentioned earlier, mosaic embryos comprise a combination of edited and unedited cells. This event first occurs when a zygote splits into a 2-cell embryo. One of the blastomeres receives sufficient amounts of GECs, and is, therefore, susceptible to gene editing via the system used in the present study, while the other blastomere might have only a few GECs that may not be sufficient to initiate genome editing at the target locus. This is particularly noticeable when low concentrations of GECs are microinjected cytoplasmically, which may hinder the formation of the sgRNA/Cas 9 complex and extend over an entire embryo, thereby leading to mosaicism [36]. Notably, Tanihara et al. [48] investigated the effects of the different concentrations of the CRISPR/Cas 9 components on the genetic mosaicism in cytoplasmically microinjected porcine embryos and reported that the ratio of the number of blastocysts that carried mutations to the total number of blastocysts examined in one group (injection at $100 \mathrm{ng} / \mu \mathrm{L}$ of each Cas9 protein and gRNA) was significantly higher than that in the other group (injection at $20 \mathrm{ng} / \mu \mathrm{L}$ of each Cas 9 protein and gRNA). Furthermore, no blastocysts with bi-allelic mutations were present in the latter group, while $16.7 \%$ of the blastocysts in the former group carried a bi-allelic mutation. In contrast, when in vitro EP was used, sufficient amounts of GECs were incorporated throughout the oocyte cytoplasm, leading to unbiased localization of the GECs upon cleavage into the 2-cell stage in mice [34]. A similar observation was reported when in vitro EP was performed in a solution containing tetramethylrhodamine-labeled dextran $3 \mathrm{kDa}$ (arrows in Figure 1B-a,b). Moreover, the present study revealed that a high efficiency (66\%; $21 / 32$ ) of obtaining the parthenotes with bi-allelic KO phenotypes could be achieved with the use of in vitro EP. These findings suggest that in vitro EP enables the unbiased incorporation of relatively higher amounts of GECs into the porcine parthenotes, which may, in turn, contribute to reducing genetic mosaicism.

Yamashita et al. [49] demonstrated the usefulness of three-prime repair exonuclease 2 (Trex2), an exonuclease that improves the gene-editing efficiency, as a reagent for suppressing mosaicism. The authors observed that the co-delivery of murine Trex2 mRNA and GECs via EP into porcine zygotes resulted in the generation of non-mosaic or mosaic mutant blastocysts with the efficiencies of $29 \%$ and $71 \%$, respectively. In contrast, the delivery of GECs alone resulted in the generation of non-mosaic or mosaic mutant blastocysts with efficiencies of $6 \%$ and $93 \%$, respectively. Therefore, this reagent could be beneficial, particularly when the in vitro EP occasionally results in poor performance in terms of inducing bi-allelic KO phenotype in the porcine embryos.

In regard to the effect of the in vitro EP on the development of porcine embryos, it was observed that there was a slight decrease in the development into blastocysts with the use of in vitro EP (30\%$38 \%$ vs. $51 \%$ ) (Table 1). Notably, Tanihara et al. [13] reported that in vitro EP may not significantly affect the blastocyst formation rates [23\% for control embryos vs. $18 \%-19 \%$ for the embryos electroporated in the presence of Cas9 mRNA + sgRNA or Cas9 protein + sgRNA]. These findings indicate the safety of using in vitro EP for the production of viable piglets. 
The off-target effects caused by the CRISPR/Cas9 system are concerning. This issue was analyzed prior to attempting the elimination of GGTA1 using the CRISPR/Cas9 system, and it was discovered that there were no off-target mutations in the candidate genes, which are theoretically prone to be genome edited[36].

Another interesting question would be whether the in vitro EP of porcine zygotes in the presence of genome editing components could be targeted toward two or more loci, as the CRISPR/Cas9based genome editing system enables targeting multiple loci in a single shot of transfection [50, 51]. Such attempts have been successful with porcine fibroblasts that were used as donor cells for the somatic cell nuclear transfer (SCNT)-mediated production of genome-edited piglets [52-55] and porcine zygotes [56]. Notably, Hirata et al. [56] investigated whether in vitro EP could be applied for one-step multiplex CRISPR/Cas9-based genome editing targeting the interleukin-2 receptor gamma (IL2RG) and growth hormone receptor (GHR) genes in porcine embryos and reported success in obtaining double bi-allelic mutations for both genes in $\sim 25 \%$ of the embryos (blastocysts). Furthermore, no significant differences in embryo development rates were detected between the control embryos and the embryos subjected to in vitro EP. This demonstrated the feasibility of using in vitro EP to transfer multiple guide RNAs and Cas9 to porcine zygotes, thereby enabling the double bi-allelic mutation of multiple genes along with favorable embryo survival.

Unfortunately, the in vitro EP-based technology for obtaining genome-edited piglets continues to be applied scarcely, with only a few laboratories worldwide reporting success in producing genome-edited porcine embryos and piglets using this technology [13-20, 56]. Tanihara and Otoi successfully knocked-out the genes encoding myostatin (MSTN) [13,20], porcine endogenous retroviruses (PERV) polymerase (pol) [18], TP53 (which encodes p53) [15], cluster of differentiation 163 (CD163) [16], and pancreas duodenum homeobox 1 (PDX-1) [17] by using in vitro EP in porcine embryos. For instance, when TP53 was targeted, all the tested blastocysts (15) exhibited mutations in the TP53 target region, while approximately $45 \%$ of the blastocysts carried bi-allelic KO mutations [15]. Notably, in our previous study concerning the in vitro EP of SCNT-treated embryos in the presence of the RNP targeted toward the low-density lipoprotein receptor ( $L D L R)$ gene, almost all $(82 \%, 9 / 11)$ of the resulting embryos (blastocysts) exhibited the bi-allelic KO genotype [14]. Taken together, these findings suggest that the in vitro EP-based genome-editing technology used in the present study is safe and efficient for the production of porcine embryos with bi-allelic mutated phenotypes.

\section{Author Contributions}

M.S conceived and designed the study, drafted the manuscript, performed the molecular analysis of parthenotes (blastocysts), and revised the manuscript. H.J. performed the experiments, especially acquisition of parthenotes, in vitro EP and embryo cultivation. E.A. and K.M. critically revised the manuscript.

\section{Competing Interests}

The authors have declared that no competing interests exist. 


\section{References}

1. Harrison MM, Jenkins BV, O'Connor-Giles KM, Wildonger J. A CRISPR view of development. Genes Dev. 2014; 28: 1859-1872.

2. Hsu PD, Lander ES, Zhang F. Development and applications of CRISPR-Cas9 for genome engineering. Cell. 2014; 157: 1262-1278.

3. Horvath P, Barrangou R. CRISPR/Cas, the immune system of bacteria and archaea. Science. 2010; 327: 167-170.

4. Bhaya D, Davison M, Barrangou R. CRISPR-Cas systems in bacteria and archaea: Versatile small RNAs for adaptive defense and regulation. Annu Rev Genet. 2011; 45: 273-297.

5. Jinek M, Chylinski K, Fonfara I, Hauer M, Doudna JA, Charpentier E. A programmable dual-RNAguided DNA endonuclease in adaptive bacterial immunity. science. 2012; 337: 816-821.

6. Gaj T, Gersbach CA, Barbas III CF. ZFN, TALEN, and CRISPR/Cas-based methods for genome engineering. Trends Biotechnol. 2013; 31: 397-405.

7. He F, Jacobson A. Nonsense-mediated mRNA decay: Degradation of defective transcripts is only part of the story. Annu Rev Genet. 2015; 49: 339-366.

8. Hammer RE, Pursel VG, Rexroad CE, Wall RJ, Bolt DJ, Ebert KM, et al. Production of transgenic rabbits, sheep and pigs by microinjection. Nature. 1985; 315: 680-683.

9. Park KW, Cheong HT, Lai L, Im GS, Kühholzer B, Bonk A, et al. Production of nuclear transferderived swine that express the enhanced green fluorescent protein. Anim Biotechnol. 2001; 12: 173-181.

10. Lai L, Kolber-Simonds D, Park KW, Cheong HT, Greenstein JL, Im GS, et al. Production of $\alpha-1$, 3galactosyltransferase knockout pigs by nuclear transfer cloning. Science. 2002; 295: 1089-1092.

11. Dai Y, Vaught TD, Boone J, Chen SH, Phelps CJ, Ball S, et al. Targeted disruption of the $\alpha 1,3-$ galactosyltransferase gene in cloned pigs. Nat Biotechnol. 2002; 20: 251-255.

12. Sheets TP, Park CH, Park KE, Powell A, Donovan DM, Telugu BP. Somatic cell nuclear transfer followed by CRIPSR/Cas9 microinjection results in highly efficient genome editing in cloned pigs. Int J Mol Sci. 2016; 17: 2031.

13. Tanihara F, Takemoto $T$, Kitagawa E, Rao S, Do LT, Onishi A, et al. Somatic cell reprogrammingfree generation of genetically modified pigs. Sci Adv. 2016; 2: e1600803.

14. Sato M, Miyoshi K, Kawaguchi H, Inada E, Saitoh I, Tanimoto A. Recent advance in genome editing-based gene modification in pigs. In Reproductive Biology and Technology in Animals. London, UK: InTechOpen; 2019.

15. Tanihara F, Hirata M, Nguyen NT, Le QA, Hirano T, Takemoto T, et al. Generation of a TP53-modified porcine cancer model by CRISPR/Cas9-mediated gene modification in porcine zygotes via electroporation. PLoS ONE. 2018; 13: e0206360.

16. Tanihara F, Hirata M, Nguyen NT, Le QA, Wittayarat M, Fahrudin M, et al. Generation of CD163edited pig via electroporation of the CRISPR/Cas9 system into porcine in vitro-fertilized zygotes. Anim Biotechnol. 2019: 1-8.

17. Tanihara F, Hirata M, Nguyen NT, Le QA, Hirano T, Takemoto T, et al. Generation of PDX-1 mutant porcine blastocysts by introducing CRISPR/Cas9-system into porcine zygotes via electroporation. Anim Sci J. 2019; 90: 55-61. 
18. Hirata M, Wittayarat M, Hirano T, Nguyen NT, Le QA, Namula Z, et al. The relationship between embryonic development and the efficiency of target mutations in Porcine Endogenous Retroviruses (PERVs) pol genes in porcine embryos. Animals. 2019; 9: 593.

19. Ongaratto FL, Rodriguez-Villamil P, Ganbaatar U, De Frutos C, Solin S, Carlson DF. 204 Efficient editing of porcine parthenogenetic zygotes by electroporation of Cas9 ribonucleoprotein complexes. Reprod Fertil Dev. 2019; 31: 227.

20. Le QA, Hirata $M$, Nguyen NT, Takebayashi $K$, Wittayarat $M$, Sato $Y$, et al. Effects of electroporation treatment using different concentrations of Cas9 protein with gRNA targeting Myostatin (MSTN) genes on the development and gene editing of porcine zygotes. Anim Sci J. 2020; 91: e13386.

21. Kaneko T, Sakuma T, Yamamoto T, Mashimo T. Simple knockout by electroporation of engineered endonucleases into intact rat embryos. Sci Rep. 2014; 4: 6382.

22. Hashimoto $M$, Takemoto $T$. Electroporation enables the efficient $m R N A$ delivery into the mouse zygotes and facilitates CRISPR/Cas9-based genome editing. Scientific reports. 2015; 5: 11315.

23. Qin W, Dion SL, Kutny PM, Zhang Y, Cheng AW, Jillette NL, et al. Efficient CRISPR/Cas9-mediated genome editing in mice by zygote electroporation of nuclease. Genetics. 2015; 200: 423-430.

24. Chen S, Lee B, Lee AY, Modzelewski AJ, He L. Highly efficient mouse genome editing by CRISPR ribonucleoprotein electroporation of zygotes. J Biol Chem. 2016; 291: 14457-14467.

25. Hashimoto $M$, Yamashita $Y$, Takemoto T. Electroporation of Cas9 protein/sgRNA into early pronuclear zygotes generates non-mosaic mutants in the mouse. Dev Biol. 2016; 418: 1-9.

26. Kaneko T. Genome editing in mouse and rat by electroporation. Methods Mol Biol.2017; 1630: 81-89.

27. Tröder SE, Ebert LK, Butt L, Assenmacher S, Schermer B, Zevnik B. An optimized electroporation approach for efficient CRISPR/Cas9 genome editing in murine zygotes. PLoS ONE. 2018; 13: e0196891.

28. Teixeira M, Py BF, Bosc C, Laubreton D, Moutin MJ, Marvel J, et al. Electroporation of mice zygotes with dual guide RNA/Cas9 complexes for simple and efficient cloning-free genome editing. Sci Rep. 2018; 8: 474.

29. Miyasaka Y, Uno Y, Yoshimi K, Kunihiro Y, Yoshimura T, Tanaka T, et al. CLICK: One-step generation of conditional knockout mice. BMC genomics. 2018; 19: 318.

30. Modzelewski AJ, Chen S, Willis BJ, Lloyd KK, Wood JA, He L. Efficient mouse genome engineering by CRISPR-EZ technology. Nat Protoc. 2018; 13: 1253-1274.

31. Nakagawa Y, Sakuma T, Takeo T, Nakagata N, Yamamoto T. Electroporation-mediated genome editing in vitrified/warmed mouse zygotes created by IVF via ultra-superovulation. Exp Anim. 2018; 67: 535-543.

32. Qin W, Wang H. Delivery of CRISPR-Cas9 into mouse zygotes by electroporation. Methods Mol Biol. 2019; 1874: 179-190.

33. Dumeau CE, Monfort A, Kissling L, Swarts DC, Jinek M, Wutz A. Introducing gene deletions by mouse zygote electroporation of Cas12a/Cpf1. Transgenic Res. 2019; 28: 525-535.

34. Sakurai T, Kamiyoshi A, Kawate H, Watanabe S, Sato M, Shindo T. Production of genetically engineered mice with higher efficiency, lower mosaicism, and multiplexing capability using maternally expressed Cas9. Sci Rep. 2020; 10: 1091.

35. Tan W, Proudfoot C, Lillico SG, Whitelaw CB. Gene targeting, genome editing: From Dolly to editors. Transgenic Res. 2016; 25: 273-287. 
36. Sato $M$, Koriyama $M$, Watanabe $S$, Ohtsuka $M$, Sakurai $T$, Inada $E$, et al. Direct injection of CRISPR/Cas9-related mRNA into cytoplasm of parthenogenetically activated porcine oocytes causes frequent mosaicism for indel mutations. Int J Mol Sci. 2015; 16: 17838-17856.

37. Kure-Bayashi S, Miyake M, Katayama M, Miyano T, Kato S. Development of porcine blastocysts from in vitro-matured and activated haploid and diploid oocytes. Theriogenology. 1996; 46: 1027-1036.

38. Jacobi AM, Rettig GR, Turk R, Collingwood MA, Zeiner SA, Quadros RM, et al. Simplified CRISPR tools for efficient genome editing and streamlined protocols for their delivery into mammalian cells and mouse zygotes. Methods. 2017; 121: 16-28.

39. 39. 55. Himaki T, Mizobe Y, Tsuda K, Suetomo M, Yamakuchi H, Miyoshi K, et al. Effect of postactivation treatment with latrunculin $A$ on in vitro and in vivo development of cloned embryos derived from kidney fibroblasts of an aged Clawn miniature boar. J Reprod Dev. 2012; 58: 398-403.

40. Lillico SG, Proudfoot C, Carlson DF, Stverakova D, Neil C, Blain C, et al. Live pigs produced from genome edited zygotes. Sci Rep. 2013; 3: 2847.

41. Sato M, Miyoshi K, Nagao Y, Nishi Y, Ohtsuka M, Nakamura S, et al. The combinational use of CRISPR/Cas9-based gene editing and targeted toxin technology enables efficient biallelic knockout of the $\alpha-1$, 3-galactosyltransferase gene in porcine embryonic fibroblasts. Xenotransplantation. 2014; 21: 291-300.

42. Ohtsuka M, Sato M, Miura H, Takabayashi S, Matsuyama M, Koyano T, et al. i-GONAD: A robust method for in situ germline genome engineering using CRISPR nucleases. Genome Biol. 2018; 19: 25.

43. Yoshioka K, Suzuki C, Tanaka A, Anas IM, Iwamura S. Birth of piglets derived from porcine zygotes cultured in a chemically defined medium. Biol Reprod. 2002; 66: 112-119.

44. Sato M, Miyagasako R, Takabayashi S, Ohtsuka M, Hatada I, Horii T. Sequential i-GONAD: An improved in vivo technique for CRISPR/Cas9-based genetic manipulations in mice. Cells. 2020; 9: 546.

45. Sato K, Yoshida M, Miyoshi K. Utility of ultrasound stimulation for activation of pig oocytes matured in vitro. Mol Reprod Dev. 2005; 72: 396-403.

46. Whitworth KM, Lee K, Benne JA, Beaton BP, Spate LD, Murphy SL, et al. Use of the CRISPR/Cas9 system to produce genetically engineered pigs from in vitro-derived oocytes and embryos. Biol Reprod. 2014; 91: 78, 1-13.

47. Burkard C, Lillico SG, Reid E, Jackson B, Mileham AJ, Ait-Ali T, et al. Precision engineering for PRRSV resistance in pigs: Macrophages from genome edited pigs lacking CD163 SRCR5 domain are fully resistant to both PRRSV genotypes while maintaining biological function. PLOS Pathog. 2017; 13: e1006206.

48. Tanihara F, Hirata M, Nguyen NT, Le QA, Hirano T, Otoi T. Effects of concentration of CRISPR/Cas9 components on genetic mosaicism in cytoplasmic microinjected porcine embryos. J Reprod Dev. 2019; 65: 209-214.

49. Yamashita S, Kogasaka Y, Hiradate Y, Tanemura K, Sendai Y. Suppression of mosaic mutation by co-delivery of CRISPR associated protein 9 and three-prime repair exonuclease 2 into porcine zygotes via electroporation. J Reprod Dev. 2020; 66: 41-48. 
50. Cong L, Ran FA, Cox D, Lin S, Barretto R, Habib N, et al. Multiplex genome engineering using CRISPR/Cas systems. Science. 2013; 339: 819-823.

51. Mali P, Yang L, Esvelt KM, Aach J, Guell M, DiCarlo JE, et al. RNA-guided human genome engineering via Cas9. Science. 2013; 339: 823-826.

52. Lutz AJ, Li P, Estrada JL, Sidner RA, Chihara RK, Downey SM, et al. Double knockout pigs deficient in $\mathrm{N}$-glycolylneuraminic acid and $\mathrm{G}$ alactose $\alpha-1,3-\mathrm{G}$ alactose reduce the humoral barrier to xenotransplantation. Xenotransplantation. 2013; 20: 27-35.

53. Li P, Estrada JL, Burlak C, Montgomery J, Butler JR, Santos RM, et al. Efficient generation of genetically distinct pigs in a single pregnancy using multiplexed single-guide RNA and carbohydrate selection. Xenotransplantation. 2015; 22: 20-31.

54. Lei S, Ryu J, Wen K, Twitchell E, Bui T, Ramesh A, et al. Increased and prolonged human norovirus infection in RAG2/IL2RG deficient gnotobiotic pigs with severe combined immunodeficiency. Sci Rep. 2016; 6: 25222.

55. Sato M, Miyoshi K, Nakamura S, Ohtsuka M, Sakurai T, Watanabe S, et al. Efficient generation of somatic cell nuclear transfer-competent porcine cells with mutated alleles at multiple target loci by using CRISPR/Cas9 combined with targeted toxin-based selection system. Int J Mol Sci. 2017; 18: 2610.

56. Hirata M, Wittayarat M, Tanihara F, Sato Y, Namula Z, Le QA, et al. One-step genome editing of porcine zygotes through the electroporation of a CRISPR/Cas9 system with two guide RNAs. In Vitro Cell Dev Biol Anim. 2020; 56: 614-621.

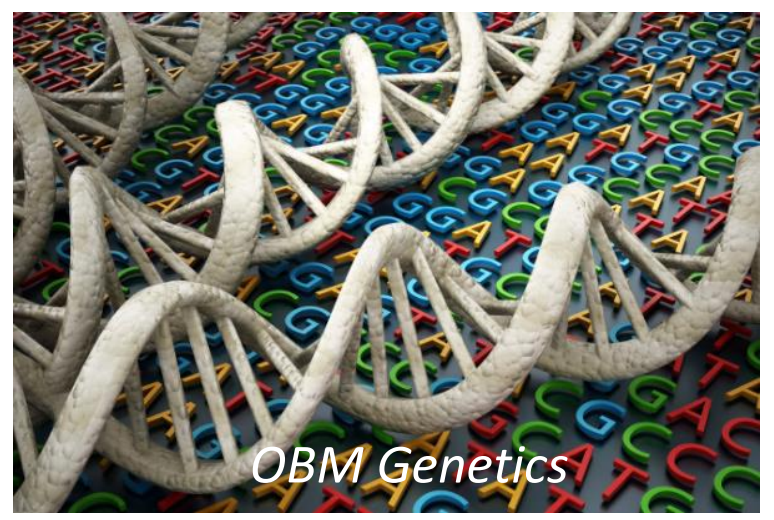

Enjoy OBM Genetics by:

1. Submitting a manuscript

2. Joining in volunteer reviewer bank

3. Joining Editorial Board

4. Guest editing a special issue

For more details, please visit: http://www.lidsen.com/journals/genetics 